\title{
Vorlesen als Impuls zur Leseförderung in der sprachlichen und kulturellen Bildung ${ }^{1}$
}

\author{
Seval Karacabey (id, Muğla \\ https://doi.org/10.37583/diyalog.1030796
}

\begin{abstract}
Deutsch)
Fremdsprachige Literatur impliziert bei Lernenden durchaus noch große Berührungsängste auch im fortgeschrittenen Stadium. Im Sinne einer sprachlichen und kulturellen Bildung sollte auf die Lektüre in der Originalsprache keinesfalls verzichtet werden. So wird insbesondere im deutschen Kulturraum das Vorlesen als Grundlage zur Entwicklung von Lesemotivation und Lesesozialisation eingesetzt. Das Vorlesen trägt in besonderer Weise zur Bildung, zur Sprachentwicklung, zur Aneignung der Kommunikations- und Ausdrucksfähigkeit bei. Durch Vorlesen kann der Zugang zur Literatur unterstützt und in unterschiedlichen Bildungsphasen gefördert werden. Untersuchungen haben nachgewiesen, dass das Vorlesen positive Auswirkungen auf die Lesemotivation und im Anschluss auf die Lesekompetenz haben kann. Wesentlich sind hier u.a. das Wissen der Lehrperson um die unterschiedlichen Vorlesephasen, sowie die Textauswahl. In diesem Beitrag geht es um die Relevanz des Vorlesens bei der Leseförderung im DaF/ DaZ-Bereich, somit in der sprachlichen und kulturellen Bildung. Als Ausgangspunkt dient in diesem Kontext ein Blick auf die Vorlesetradition in der türkischen und deutschen Gesellschaft, um auf die unterschiedlichen Entwicklungen in der Vorlesekultur hinzuweisen. In diesem Rahmen liegt der Fokus auf der Frage: Wie kann die Literalität im Fremdsprachenunterricht eingesetzt werden, um das Erlernen einer Sprache zu unterstützen? Darauf aufbauend werden Erfahrungen von Lehrpersonen und empirische Ergebnisse zum Vorlesen in der deutschen Gesellschaft thematisiert und erläutert und einige Konzepte vorgestellt. Ausgehend davon werden schließlich Überlegungen und Möglichkeiten für die Praxis, sowie konkrete Tipps zur Umsetzbarkeit im DaF/ DaZ-Bereich am Beispiel von Uwe Timms Kinderroman „Die Zugmaus“ diskutiert. In diesem Roman nimmt eine Maus die Leser auf eine Reise durch unterschiedliche Länder mit. Dadurch ist das Buch abwechslungsreich und spannend zugleich und zum Vorlesen besonders geeignet. Dies kann als Zeichen dafür gewertet werden, dass das Vorlesen als Leseförderungsmaßnahme für Jugendliche und junge Erwachsene auch im Studium Freude bereiten kann.
\end{abstract}

Schlüsselwörter: Kinderroman, Literatur, Literalität, Literarisches Lernen, Leseförderung Durch Vorlesen.

\section{Abstract (English)}

\section{Reading aloud as an impulse to promote reading in language and cultural education}

Foreign language literature still implies great reservations among learners, even at an advanced stage. In the interests of linguistic and cultural education, reading in the original language should by no means be dispensed with. In particular, in the German cultural area, reading aloud is used as a basis for developing

Einsendedatum: 28.10.2021

Freigabe zur Veröffentlichung: 01.12.2021

${ }^{1}$ Ein Teil dieser Artikel wurde als Tagungsbeitrag auf dem XV. Internationalen Türkischen Germanistik Kongress an der Universität Trakya in Edirne, der vom 30. September bis 02. Oktober 2021 Online organisiert wurde, vorgetragen. 
reading motivation and reading socialization. Reading aloud contributes in a special way to education, language development and the acquisition of communication and expression skills. The reading process can support access to literature and promote it in different educational phases. Studies have shown that reading aloud can have positive effects on reading motivation and, subsequently, on reading skills. Essential here are, among other things, the knowledge of the teacher about the different reading phases and the choice of text. This article deals with the relevance of reading in promoting reading in $\mathrm{DaF} / \mathrm{DaZ}$, thus in linguistic and cultural education. In this context, the starting point is a look at the reading aloud tradition in Turkish and German society in order to point out the different developments in the reading culture. In this sense, the focus is on the question: How can literacy be used in foreign language teaching to support the learning of a language? Building on this, the experiences of teachers and empirical results on reading aloud in German society are discussed and explained and some concepts are presented. Based on this, considerations and possibilities for practice, as well as concrete tips on how to implement them in $\mathrm{DaF} / \mathrm{DaZ}$, are discussed using the example of Uwe Timm's children's novel „Die Zugmaus“. In this novel, a mouse takes the reader on a journey through different countries. This makes the book varied and exciting at the same time and particularly suitable for reading aloud. This can be seen as a sign that reading aloud as a reading promotion measure can also be fun for adolescents and young adults during their studies.

Keywords: Children's Novel, Literature, Literacy, Literary Learning, Reading Promotion Through Read Aloud. 


\section{EXTENDED ABSTRACT}

Reading aloud contributes in a special way to education, language development and the acquisition of communication and expression skills. The reading aloud process can support access to literature and promote it in different educational phases. Studies have shown that reading aloud can have positive effects on reading motivation and, subsequently, on reading skills. Essential here are, among other things, the knowledge of the teacher about the different reading phases and the choice of text. In this context, the starting point is a look at the reading aloud tradition in Turkish and German society in order to point out the different developments in the reading culture. In this sense, the focus is on the question: How can literacy be used in foreign language teaching to support the learning of a language? Building on this, the experiences of teachers and empirical results on reading aloud in German society are discussed and explained and some concepts are presented. Based on this, considerations and possibilities for practice, as well as concrete tips on how to implement them in $\mathrm{DaF} / \mathrm{DaZ}$, are discussed using the example of Uwe Timm's children's novel "Die Zugmaus". In this novel, a mouse takes the reader on a journey through different countries. This makes the book varied and exciting at the same time and particularly suitable for reading aloud. This can be seen as a sign that reading aloud as a reading promotion measure can also be fun for adolescents and young adults during their studies. Regular reading aloud has a positive effect on foreign learners. Reading aloud is intended to increase reading motivation and arouse curiosity about literature. The text selection must be well thought out. The text should be funny, interesting, appealing, light and exciting. Teachers also act as reading models, so the teacher should emphasize well, read aloud in an understandable manner and establish eye contact with students. Facial expressions, gestures and voices play an important role.

Reading aloud in foreign language lessons can have a learning-promoting effect for beginners and advanced learners in a university context and can be practiced as a learning activity or reading strategy. However, the awareness of reading aloud as a reading strategy and its role in foreign language learners is immense. In particular, students with poor reading skills could learn about literature by reading aloud, reflect on it and talk about it and, last but not least, discover the pleasure of reading in the foreign language. Reading and listening to a story in language and literature-oriented foreign language lessons is an important point that should be in the foreground, because reading aloud can introduce literacy. By reading aloud regularly, the students can practice pronunciation and intonation and get the impetus to read aloud themselves. When reading this children's novel "Die Zugmaus", the aim is to give the students the motivation to read and the courage to read foreign-language literature. Children's novels are recommended for reading aloud, as this medium promotes several areas of competence, such as the reading and writing skills of the students. Own and foreign living environments can be discovered and new perspectives can be explored. Learners should also come into contact with reading aloud in the foreign language at the university level. Through children's literature in this context, suitable reading situations can also be created in the university context. The images that accompany the children's novel can induce reading conversations. One can present a literary text in different ways. For example, one can design the reading situations with questions to promote language. Asking questions about the text is a proven strategy. It stimulates learners to think and speak and offers them the opportunity to participate actively in the interaction with the text. The "feedback conversation" and the "literary conversation" are also suitable forms of literary communication that can be used in the context of foreign language reading. Reading aloud activities can also be organized for this purpose. Voluntary reading mentors could also strengthen reading aloud in everyday university life and regular reading hours and reading competitions could be incorporated into the course. 
However, the meaning and purpose of reading aloud as a literacy activity should be made aware of the German teacher training candidates and opportunities or "follow-up communication" should be created to act linguistically. 


\section{Einleitung}

Vorlesen gehört zu den wichtigsten Faktoren, die Literalität ausbilden und sich auch auf die schriftsprachlichen Kompetenzen der Kinder auswirken. Auch im Bereich DaF/ DaZ ist die Einbindung von Vorlesen ein Kriterium zur Entwicklung der Literalität von Lernenden (vgl. Lundquist-Mog / Widlok 2015: 57). So gilt das Vorlesen ,als immens wichtige Literacy-Erfahrungen in der frühen Kindheit" (Nickel 2007: 23). Es wurde belegt, dass das Vorlesen nicht nur Auswirkungen auf die Entwicklung der Lese- und Schreibkompetenz der Kinder hat, sondern auch auf die mündliche Ausdruckskompetenz. Kindern, denen regelmäßig vorgelesen wurde, konnten deutlich kompetenter mit der Sprache umgehen (vgl. ebd.). Folgendes ist in diesem Sinne besonders hervorzuheben: „Literalität beginnt lange vor dem Lesen und beinhaltet alles rund um die Schriftkultur. Das beginnt mit dem Zuhören beim Vorlesen und dem Bilderbuch [...]“(ebd.). An dieser Stelle ist die Aussage von Huneke und Steinig erwähnenswert: „Ohne hörendes Verstehen ist Sprachenlernen kaum vorstellbar - der größte Teil des sprachlichen Inputs, anhand dessen Sprachlerner die Fremdsprache erwerben, begegnet ihnen als gesprochenes Wort“" (2002: 117). In der Vorlesesituation hat das (Zu-)Hören eine zentrale Bedeutung. Das Vorlesen von interessanten Texten der Kinder- und Jugendliteratur stellt auch „ein sehr motivierendes Verfahren für die Leseförderung dar“ (Nickel 2007: 109). Das Vorlesen ist eine Methode, die sowohl in Familien als auch in institutionellen Einrichtungen wie Kindergärten und Schulen genutzt wird. Es wird als ein Interaktionsformat bezeichnet und wie folgt beschrieben:

\footnotetext{
Es hat den Stellenwert eines Sprachlernsettings, das Kinder im Spracherwerb vielseitig unterstützt, ihre kognitive Entwicklung von frühen Kindheitsjahren an fördert, literarische Fähigkeiten anbahnt und den Schriftspracherwerb vorbereitet. [...] Der Vorleser, das Kind und das Buch stehen dabei in einer Wechselbeziehung zueinander, die den kontexttuellen Rahmen der Situation bestimmt. (Gressnich / Müller / Stark 2015: 7)
}

Die Komponenten des Vorlesens wie der Vorleser, das Buch und das Kind werden mit dem Sprach- und Literaturerwerb in Beziehung gesetzt. Es findet sozusagen eine Vorleseinteraktion statt. In diesem Kontext spielt die Auswahl der Bücher zum Vorlesen eine besondere Rolle, denn inhaltliche, narrative Eigenschaften des Werkes können mehrere Fähigkeiten anregen. Ausschlaggebend ist hier die Buchgrundlage und die Vorleser-Kind-Interaktion. Das Text-Bild-Verhältnis sollte allerdings in Betracht gezogen werden. Das Vorlesen fördert somit sprachliches und literales Lernen. Literale Fähigkeiten können bereits in der frühkindlichen Entwicklung gefördert werden. Dieses wird in der Interaktion zwischen Interaktionspartner und Kind ermöglicht. Das Vorlesen wird als eine Diskurs- und Sprachaktivität bezeichnet, die in der westlichen Kultur eine fundamentale Rolle in der kindlichen Sprachsozialisation spielt. Das Vorlesen als „Lernmedium“ hat den Stellenwert, dass die Vorlesesituation durch den Vorleser interaktiv gestaltet werden kann (vgl. Becker / Müller 2015: 79ff). Das Vorlesen im engen Sinn bedeutet also das laute Lesen eines Buches, ,wobei ein Leser einem oder mehreren Zuhörern vorliest. Weiteren soll auch die unmittelbare Verbalisierung einer illustrierten oder nicht illustrierten Textgrundlage verstanden, die eine globale narrative Struktur aufweist." (Becker / Müller 2015: 81) Von Vorlesen im weiten Sinn ist die Rede, wenn 
der Vorlesende den Text durch eigene Anmerkungen ergänzt. Bilderbücher stellen eine Kombination von Bild und Text dar. Hier wird das Bildmaterial beim Vorlesen interpretiert (Becker / Müller 2015: 81). Dieser Prozess wird wie folgt ausgeführt: „Sprachaktivitäten können Kommentierungen sein, die sich auf die Illustrationen im Buch beziehen oder auf die außertextuelle Welt verweisen mit der Funktion das Kind in den Vorleseprozess stärker einzubeziehen sowie sein Text- und Bildverständnis zu unterstützen“ (ebd.). Als wichtige charakteristische Merkmale des Vorlesens fassen Becker und Müller wie folgt zusammen: das Vorlesen kann nur im Mündlichen realisiert werden. Es erfordert starke Textbasiertheit, einen größeren Bedarf an interaktiver Anpassung und die Gebundenheit an das Medium Buch, sowie ein differenzierteres Vokabular aufgrund der stärkeren Textbasiertheit (vgl. ebd.: 90).

\section{Empirische Studien zum Vorlesen als Leseförderung}

Die empirischen Untersuchungen vermitteln zahlreiche positive Effekte des Vorlesens. Ausgehend der Untersuchungen bereitet das Vorlesen Freude und unterstützt die Sprachentwicklung und Sozialkompetenz. Das Vorlesen „fördert die kognitiven emotionalen sowie sozialen Kompetenzen und trägt dazu bei, dass Kinder und Jugendliche sich zu vielseitig interessierten, aktiven und offenen Menschen entwickeln. “2 Der Einfluss des Vorlesens in der Familie und der Schule hat große Wirkung auf das Lernen und auf die Lesekompetenz. In diesem Kontext berichten Belgrad und Klippstein in Anlehnung an Studien von den positiven Auswirkungen des Vorlesens auf die Lesemotivation und die Lesebereitschaft der Schüler und stellen die elementare Schlüsselrolle des Vorlesens für die Leseförderung heraus (vgl. Belgrad / Klippstein 2015: 180f). Laut Belgrad und Klippstein erfüllt das Vorlesen sechs Funktionen: Eine kulturelle, eine literarisch-ästhetische, eine kognitive, eine emotionale, eine kommunikative und eine reflektive Funktion. Um einen differenzierten und fachlichen Blick auf das Vorlesen zu erhalten, soll in diesem Beitrag auf die sechs wesentlichen Funktionen des Vorlesens näher eingegangen werden:

1. Die kulturelle Funktion; hierbei geht es beim Vorlesen ,um eine Weitergabe einer kulturellen und literarischen Tradition [...]. Vorlesen ermöglicht das Erfahren konzeptioneller Schriftlichkeit im Medium des Mündlichen.“(ebd.: 181f.)

2. Die literarisch-ästhetische Funktion: Die Lernenden setzen sich intensiv mit literarischen Figuren auseinander. In Anlehnung an Garbe / Holle / Weinhold (2011:7) schreiben Belgrad und Klippstein, dass Lehrkräfte Lesevorbilder und damit faktisch Lesemodelle für Lernende sein können, an denen sich diese orientieren können (vgl. Belgrad / Klippstein 2015: 182).

3. Die kognitive Funktion: Hier wird ausschließlich zugehört. Lernende müssen nur zuhören und nicht lesen. Die Absicht ist die Schulung des Zuhörens und des Verstehens. Auf diese Weise sollen auch innere Vorstellungsbilder erzeugt, das Zuhören geschult und das Verstehen gefördert werden (vgl. ebd.).

\footnotetext{
${ }^{2}$ URL: www.vds-ev-sachsen-anhalt.de (Letzter Zugriff: 30.07.2021).
} 
4. Die emotionale Funktion: Die Lernenden sollen neben Schaffung einer Leseatmosphäre, die durch positives Leseklima unterstützt wird, für Literatur begeistert werden. Dabei bildet sich nicht nur die Faszination für das Vorgelesene, sondern auch „das Einfühlen in die literarischen Figuren und eine mögliche Perspektivübernahme der emotionalen Haltungen der Figuren“ aus (vgl. Belgrad / Klippstein 2015: 183).

5. Die kommunikative Funktion: Hier können die Lernenden über das Vorgelesene ins Gespräch kommen. Sie können dabei mit der „Anschlusskommunikation“ angeregt werden, über den Text nachzudenken (vgl. Belgrad u.a. 2015: 183). Im Hinblick auf die Gespräche im Zusammenhang mit dem Vorlesen wird Folgendes ausgeführt:

\footnotetext{
Diese Form des Gesprächs, das vor, während oder nach dem Vorlesen erfolgt, ist ein wichtiger motivationaler Faktor, um die Lernenden anzuregen, sich über das Gehörte auszutauschen. Durch Gespräche über das Vorgelesene verbessern die Lernenden ihren mündlichen Ausdruck, indem sie das beim Zuhören Empfundene verbalisieren, Leerstellen füllen, Wortbedeutungen erschließen und dadurch ihren Wortschatz erweitern. Sie erhalten durch das Vorlesen ein Vorbild für sprachliche Korrektheit im Hinblick auf Aussprache, Intonation und Grammatik. (Belgrad / Klippstein 2015: 183)
}

Ausgehend von dieser Erkenntnis, erscheint das Vorlesen besonders effektiv beim Erlernen einer Fremdsprache und bei der Förderung der mündlichen Kommunikationskompetenz zu sein. Das Allerwichtigste ist, dass das Vorlesen aus Phasen (vor-während-nach) besteht.

6. Die reflexive//reflektive Funktion: Die Lernenden reflektieren hier über die Erfahrungen der literarischen Figuren. Sie setzen sich mit den Lebensentwürfen von Figuren auseinander, die die Lernenden zum Nachdenken und zum Betrachten eigener Lebenssituationen und -gestaltung führen. Folgendes ist in diesem Punkt besonders hervorzuheben:

\footnotetext{
Erleben die Kinder eine Vielfalt von literarischen Begegnungen, so erleben sie auch eine Vielzahl von Lebensentwürfen, die ihr Potenzial an Lebensgestaltungen erweitern. Kommen noch mündliche und schriftliche Prozesse in der Anschlusskommunikation oder in literarischen Gesprächen hinzu, befinden wir uns im Übergang zum ,Reflektieren und Bewerten'. (Belgrad / Klippstein u.a. 2015: 183)
}

Belgrad und Klippstein stellen fest, dass die Ergebnisse aller Studien von 2010-2015 in eine Richtung zeigen und ziehen den Schluss, dass die basalen Lesefähigkeiten sich durch regelmäßes Vorlesen entwickeln. Ausschlaggebend ist, dass der Effekt des Vorlesens steigt, wenn Lehrkräfte geschult wurden, wenn Lehrkräfte leseschwächeren Schülern nur vorlasen und diese nicht mitlesen mussten, wenn die Lehrperson mit der Klasse im Anschluss an das Vorlesen über den Text redete. Als weitere Effekte fügen sie hinzu, dass durch Vorlesen die Klassengemeinschaft gestärkt wird und die Arbeitsatmosphäre in der Klasse sich verbessert. Des Weiteren nimmt die Konzentrationsfähigkeit der Schüler zu. Darüber hinaus nimmt das Interesse an Büchern und die Lust am SelberLesen zu (vgl. ebd.: 195f.). In diesem Zusammenhang gehört das Vorlesen zum Alltag der deutschen Sprache und Lesekultur. Dies belegen die Vorlesestudien. Man stößt sehr viele Projekte an, beispielsweise beim ,der bundesweite Vorlesetag“ (2014/ 2015), die das Thema Vorlesen in den Vordergrund stellen und wissenschaftlich belegen, dass mit Vorlesen sehr viele Kompetenzen gefördert werden können und zum Schulerfolg 
beitragen können. So führen die Studien (2013/ 2015) zum Vorlesen folgende positiven Ergebnisse aus ${ }^{3}$ :

- Vorlesen und späterer Schulerfolg hängen eng zusammen. [...],

- Kinder, denen vorgelesen wurde, gehen besonders gern zur Schule [...],

- Vorlesen steht in Zusammenhang mit guten Voraussetzungen für Lern- und

Schulerfolg [...],

- Vorlesen steht in Zusammenhang mit guten Voraussetzungen für den 'Ernst des

Lebens' $[\ldots]$,

- Faktoren für bessere Leistungen sind Wissbegierde, Freude am Lernen, kognitive

Stärken und Ausdauer. [...],

- Kinder, denen vorgelesen wurde, haben einen weiten Horizont [...],

- Kinder, denen vorgelesen wurde, sind besonders gern mit anderen zusammen [...],

- Kinder, denen vorgelesen wurde, sind für die anderen mehr als nur Spielkameraden [...],

- Gerade sozial eher isolierte Kinder profitieren vom Vorlesen für den Umgang mit anderen $[\ldots]$,

- Sensibilität, Empathie und Interesse für andere sind typisch für Kinder, denen vorgelesen wurde [...],

- Kinder, denen vorgelesen wurde, zeigen sich besonders zupackend und engagiert $[\ldots]$,

- Vorlesen leistet einen wichtigen Beitrag zur emotionalen Stärke und zur sozialen

Kompetenz. [...],

- Vorlesen stärkt Kinder in ihrer persönlichen Entwicklung. [...],

- Vorlesen stärkt soziale Beziehungen. [...],

- Kinder, denen vorgelesen wurde, zeigen bereits gesellschaftliche Verantwortung

$[\ldots]$,

- Vorlesen stärkt gesellschaftliches Miteinander [...],

- Eltern stärken die Zusammenhänge zwischen Vorlesen und sozialer Kompetenz, indem sie das Vorlesen als Gesprächsanlass und zur Verarbeitung von Fragen, Problemen, Sorgen etc. nutzen. [... $]^{4}$

\section{Zur Tradition des Vorlesens in der deutschen und türkischen Gesellschaft}

Deutschland hat eine besondere Vorlesekultur. Insbesondere ist das Vorleseverhalten in der Familie in Deutschland sehr prägend, während dies im türkischen Kontext sowohl in der häuslichen als auch in der schulischen Umgebung nicht der Fall ist. Die Vorlesekultur ist in der deutschen Sprache und Lesekultur fest verankert, dennoch belegen Untersuchungen, dass den Kindern aus bildungsfernen Familien weniger vorgelesen wird. Laut einer Vorlesestudie der Stiftung Lesen von 2017, realisiert durch „Die Zeitund die Deutsche Bahn Stiftung", kann gesagt werden, dass in Familien Lesen und Bildung als besonders wichtig angesehen werden, obwohl Eltern relativ spät mit dem Vorlesen beginnen. Es gibt in Deutschland Bildungseinrichtungen, Akademien für Leseförderung, Programme zur Leseförderung in der Familie, die sich für die Entwicklung/ Verbreitung der Leseförderung einsetzen und dabei die Eltern informieren

\footnotetext{
${ }^{3}$ URL: https://www.familienservice.unimainz.de/files/2013/10/VLStudie_2015_Website_20151118_03.pdf (Letzter Zugriff: 10.10.2021).

${ }^{4}$ URL: https://www.familienservice.unimainz.de/files/2013/10/VLStudie_2015_Website_20151118_03.pdf (Letzter Zugriff: 30.09.2021).
} 
und für das frühe Vorlesen gewinnen. Des Weiteren gibt es ein bundesweites Leseförderprojekt und es werden Vorleseaktionen veranstaltet. Neben der Familie sind Krippen und Kitas wichtige Institutionen in der frühkindlichen Leseförderung. Darüber hinaus gibt es Vorleseangebote in Stadtbibliotheken oder ehrenamtliche Vorlesepatinnen und -paten. ${ }^{5}$ Zudem ist besonders bemerkenswert, dass Vorlesetage, ehrenamtliche Vorleser, Literaturfestivals, Autorenlesungen, Literaturtage, Lesefeste, Wettbewerbe und Literaturhäuser wichtige Plattformen sind, wo das Vorlesen als lesefördernde Methode praktiziert wird. Während im deutschsprachigen Raum das Vorlesen eine weit verbreitete Methode der Kulturförderung ist, wird das Vorlesen in der Türkei laut Forschungsberichten sehr geringgeschätzt. Dies hängt stark mit dem Bildungsniveau der Familie, mit dem kulturellen und sozio-ökonomischen Status zusammen. Die Kultur des Geschichtenerzählens in Form der mündlichen Erzählkultur ist in den türkischen Familien dominanter als die schriftlich fixierte Lesekultur. Der mindere Wert des Lesens, das Bewusstsein über die Vorbildfunktion des Lesens und die fehlende Geduld zum Vorlesen werden als Gründe aufgeführt. Erst die neueren Forschungen in diesem Bereich belegen, dass das Vorlesen als effektive Methode der Sprachförderung seine Berechtigung hat. Die Auswirkungen des Vorlesens der Eltern auf die sprachliche Förderung der Kinder im Vorschulalter und im Primarbereich wird stark hervorgehoben, da man sich die Lesegewohnheit im frühen Alter aneignet (vgl. Güneş 2011; Ergül u.a. 2015). Des Weiteren beobachtete Kuyumcu (2008) in seiner Forschung, dass das Vorlesen in türkischen Familien unzureichend ausgeprägt ist, dass nur wenige Mütter und Väter ihren Kindern Geschichten aus Büchern vorlesen.

\section{Kinder und Jugendliteratur zum Vorlesen}

,Das literarische Lernen', ,der Aufbau und Sicherung von Lesemotivation', ,Vermittlung von Lesefreude und Vertrautheit mit Büchern', ,Entwicklung und Stabilisierung von Lesegewohnheiten“ und ,Förderung der Lesefähigkeit" werden als die zentralen Aufgaben des Literaturunterrichts beschrieben (vgl. Paefgen 1999: 88). Es sollten Lesesituationen entwickelt werden, in denen Lesekompetenzen und literarische Rezeptionskompetenzen erworben werden können. Diese sollten die Möglichkeit bieten vielfältige Erfahrungen mit Literatur zu machen (vgl. Gölitzer 2010: 222). Ausgehend von diesen Feststellungen ist die Bedeutung der Kinder- und Jugendliteratur in der literarischen Sozialisation, d.h. in den Familien, den Vorschuleinrichtungen, in der Schule und im Studium, immens. Über Kinderliteratur wird Folgendes ausgeführt: „Das Potential der Kinderliteratur eröffnet die Förderung einer Reihe von Fähigkeiten und Kompetenzen im Fremdsprachenunterricht. Dazu gehören u.a. Literalität, narrative und kommunikative Kompetenz" (Ciepielewska-Kaczmarek 2016: 87f.). Außerdem bietet das Vorlesen literarischer Texte vielfältige Möglichkeiten. Die literarische Kommunikation lässt sich unter dem Begriff „Anschlusskommunikation“ zusammenfassen. Diese wird als „Kommunikation“ bezeichnet, die sich an den Text ,anschließt‘, ,also den Text kommentiert, ihn befragt, sich mit ihm auseinandersetzt:

\footnotetext{
${ }^{5}$ URL: www.nifbe.de (Letzter Zugriff: 15.10.2021).
} 
davor-während-nach dem Vorlesen“ (Belgrad / Schattmann 2015: 1). Dabei gibt es die folgenden Interaktionsformen der Anschlusskommunikation: Interaktives Vorlesen, Feedback-Gespräch, Literarisches Gespräch (vgl. ebd.). Auch die Kinder- und Jugendliteratur kann im Kontext des Vorlesens und der literarischen Erziehung zum literarischen Lesen/ Lernen Entwicklungen anbahnen, die für die Kinder und Jugendlichen lebensbestimmend sind. Die Beschäftigung mit Kinder- und Jugendliteratur ist für Fremdsprachenlerner auch im Hochschulkontext, sowie für angehende Deutschlehramtskandidaten außerordentlich wichtig, da sie neben der sprachlichen auch eine fachliche Ausbildung bekommen sollten. Für die Studierenden bietet sich somit auch die Gelegenheit, sich im Bereich der Kinder- und Jugendliteratur zu orientieren und diese in ihre spätere Lehrtätigkeit zu integrieren. Im Kontext des Vorlesens von Kinder- und Jugendliteratur unterstreichen Rosebrock und Nix die Rolle des Vorlesens von Büchern im Prozess der Lesesozialisation wie folgt:

[D]a Kinder dadurch bereits vor dem Erwerb der Lese- und Schreibfähigkeit mit grundlegenden Erzählstoffen und -strukturen und mit situationsabstrakter Sprache vertraut gemacht werden. Auch für die spätere Schulzeit stellt das Vorlesen von interessanten Texten der KJL ein sehr motivierendes Verfahren für die Leseförderung dar. (2012: 109)

Die Erzählung „Die Zugmaus“ von Uwe Timm eignet sich sowohl inhaltlich als auch sprachlich besonders gut zum Vorlesen auch im fremdsprachlichen Rahmen, aber auch als Anschlusskommunikation nach dem Vorlesen kann man rezeptiv und produktiv gut damit arbeiten. Des Weiteren gibt es zahlreiche interkulturelle und universelle Themen, die zu literarischen Gesprächen im fremdsprachlich orientierten Literaturunterricht im universitären Kontext führen könnten. Auch das Betrachten der Bilder im Kinderroman „Die Zugmaus“ bietet sprachfördernde, leseförderliche Angebote für den Fremdsprachenlerner. Wobei der Bildbetrachtungsprozess auch durch Fragen unterstützt werden kann. In der Erzählung „Die Zugmaus“ von dem bekannten Schriftsteller Uwe Timm nimmt die Maus mit dem Namen Stefan die Leser auf eine Reise durch unterschiedliche Länder mit und lernt das Käseparadies Schweiz und die BaguetteHauptstadt Paris kennen. Mit einem Zirkuszug gelangt Stefan sogar nach England. Unterwegs lernt er Menschen, ländertypische Spezialitäten kennen und findet Freunde. Nach drei Jahren bekommt Stefan Heimweh und kehrt nach Hause zurück. Die Hauptfigur, eine Maus erzählt ihre abenteuerliche Reise. Doch zuvor stellt die Maus zunächst sich und ihren Wohnort und danach ihre Familie vor. Dabei verspricht sie dem Leser/ Zuhörer, dass es in ihrer Geschichte um real existierende Orte, Plätze, Städte und Länder geht. Der Beginn des ersten Kapitels lautet:

,Wo warst du denn die ganze Zeit?‘ ,Bist du wirklich nach Paris gekommen?` ,Und wo hast du dich im Zug versteckt?' So werde ich immer wieder gefragt und muss jedes Mal meine Abenteuer erzählen.

[...]

Ich bin eine gewöhnliche Hausmaus und heiße Stefan.

$[\ldots]$

Geboren wurde ich in München, und zwar in der Paradiesstraße. 
Wer jetzt glaubt, dieser Straßenname sei eine Erfindung von mir, der soll sich einen

Münchner Stadtplan besorgen und darin die Straße suchen.

Die Straße gibt es wirklich. Nur mein Geburtshaus steht leider nicht mehr. (Timm 2007:

5f.)

Dieser Kinderroman zeichnet sich auch durch internationale und interkulturelle Bilder aus: „Hoffentlich hält der Zug auch wirklich in München“, sagte ich. „Stell dir vor, wir fahren nach Istanbul, das liegt doch irgendwo in der Türkei“" (Timm 2007: 100). Auf der Rückreise im Zugabteil beschreibt die Maus auf der Rückfahrt von Hamburg nach München eine Familie. So geht es im Kapitel 20 um eine griechische Familie, der die Maus (Stefan) im Zugabteil begegnet. Diese Begegnung beschreibt die Maus folgendermaßen:

\begin{abstract}
Wir schlichen uns durch den Zug. Alle Abteile waren voll. Schließlich fanden wir eins, in dem eine griechische Familie saß, Vater und Mutter mit vier Kindern. Der Vater und die Mutter hatten drei Jahre in Hamburg gearbeitet, er bei der Müllabfuhr, sie als Putzfrau. Jetzt kehrten sie nach Griechenland zurück. Sie hatten das ganze Abteil mit Kästen und Kartons vollgestellt, und in einem Korb, oben auf dem Gepäcknetz, gackerten sogar zwei Hühner. (Timm 2007: 100ff.)
\end{abstract}

Uwe Timm lenkt den Blick auf verschiedene Perspektiven und andere Kulturen. Dieser Kinderroman ist reich an Selbst- und Fremdbildern, und dadurch eignet sich dieser Roman nicht nur zum Vorlesen im fremdsprachlichen Kontext, sondern auch für interkulturell orientierte Vorlesegespräche. Ziel des Vorlesens aus dem Kinderroman „Die Zugmaus“ ist es u.a. auch, Interesse und Neugier für Literatur und für fremde Kulturen $\mathrm{zu}$ wecken. Und auch einen sprachlichen und kulturellen Kontakt durch Vorlesen herzustellen. Der Kinderroman „Die Zugmaus“ von Uwe Timm wurde im Hinblick auf seine inhaltlichen Elemente für den Leselernprozess in einem Seminar vorgelesen und kommentiert. Das Ziel dabei war das Vorlesen zu thematisieren, damit die angehenden Deutschlehramtskandidaten angeregt werden, im Berufsleben diese Strategie zu nutzen. Insbesondere Kinder- und Jugendliteratur kann zum Vorlesen und für das Training von Aussprache, Intonation, aber auch zur Aneignung von literarischen und (inter-)kulturellen Kompetenzen genutzt werden. Schließlich sollten angehende Deutschlehramtskandidaten über Wissen zur Literatur für Kinder in der zur vermittelnden Fremdsprache verfügen. Auch lernen sie dabei, das Vorlesen als Methode der Sprachvermittlung zu nutzen. Der Kinderroman von Uwe Timm bietet Raum für ,literale Anschlusskommunikationen“ im interkulturellen Vorleseunterricht. Man kann über Literatur, den Textinhalt, über das Eigene und das Fremde verschiedener Kulturen reden. So werden neben sprachlichen und kulturellen Aspekten auch universelle Daseinserfahrungen wie Freundschaft, Heimweh, Angst, Mut, Trennung, Nachbarschaft, Tierliebe, Umwelt, Reisen, Familie, Zusammenhalt und Neugier usw. thematisiert. Durch all diese Themen ist der Roman abwechslungsreich und spannend und zum Vorlesen und zur Gestaltung von „Anschlusskommunikation“ besonders geeignet. Zudem sind sehr viele Zeichnungen, Bilder und Dialoge in diesem Kinderroman enthalten und er umfasst 23 kurze Kapitel, die unterschiedliche Vorlese- bzw. Lesesituationen ermöglichen können. Im Folgenden soll es nur um eine beispielhafte Beschreibung von VorleseLesesituation bzw. Vorleseerfahrung im Studium gehen. Die Studierenden bekamen 
Textauszüge (Insgesamt 5 Kapitel) aus dem Kinderbuch „Die Zugmaus“ durch die Lehrperson vorgelesen. Die Lehrperson las den Text neutral vor. Die Studierenden hörten nur zu. Hierbei ist zu vermerken, dass sich einige Studierende gemeldet haben und bekanntgaben, dass sie beim Zuhören gern mitgelesen hätten. Das Vorlesen erfolgte per Online-Unterrricht. Folgende Leseaufgaben wurden nach dem Vorlesen als rezeptive und produktive Leseaufgaben im Sinne einer „literalen Anschlusskommunikation“ bearbeitet:

- Lesen Sie die Kapitel 1 bis 5 aus dem Kinderroman „Die Zugmaus“ von Uwe Timm.

- a. Schreiben Sie 3 Lieblingsstellen aus den 5 Kapiteln heraus. Warum haben Ihnen diese Textstellen besonders gut gefallen? Begründen Sie.

- b. Welche Textstellen sind Ihrer Meinung nach die drei am schönsten

formulierten aus den 5 Kapiteln. Schreiben Sie diese Formulierungen aus den 5

Kapiteln heraus und äußern Sie sich zum Inhalt, zum Stil und zur benutzten

Sprache.

Literarische Gespräche und Feedback-Gespräche als „literale Anschlusskommunikation“ eignen sich besonders gut für die Phase nach dem Vorlesen im fremdsprachlichen Literaturunterricht an höheren Bildungseinrichtungen.

\section{Welche Rolle spielt das Vorlesen in der Fremdsprache im Hochschulkontext?}

Die Vorleseerfahrungen, sowohl in der Mutter- als auch in der Fremdsprache der türkischen Deutschlehramtskandidaten sind sehr gering, weil das Vorlesen in Familien und auch im schulischen Umfeld keine Tradition besitzt und nicht gepflegt wird. Dennoch wird bei der Auswertung von Fragebögen vorangegangener Studien eine positive Einstellung zum Vorlesen bei den Deutschlehramtskandidat*innen deutlich. Hier ist zu bemerken, dass im Seminar nicht kontinuierlich vorgelesen wurde. Das Vorlesen wurde nur als Lesestrategie/ Lesetechnik zur Förderung der fremdsprachlichen Lesekompetenz eingesetzt. Ausgehend von der Untersuchung im Hinblick der angewendeten Lesestrategien/ Lesetechniken stellte sich heraus, dass sich die Vorlesemethode als eine beliebte Tätigkeit unter den weiteren angewendeten Lesestrategien aus der Perspektive der Studierenden erwies. Die Mehrheit (45 von 49) der Deutschlehramtskandidat*innen werteten das Vorlesen als sehr positiv 6 . Das Vorlesen als Methode auf der Hochschulebene in der Fremdsprachenausbildung sollte regelmäßig gepflegt konsequent weitergeführt und auf einem höheren Sprachniveau möglichst oft eingesetzt werden. Studierenden wünschen sich das Vorlesen, weil es u.a. auch das Hörverstehen trainiert und der Fokus auf Artikulation, Aussprache, Stimme, Ton und Intonation liegt, was eine bedeutende Rolle für angehende Deutschlehramtskandidaten beim Fremdsprachenlernen spielt. Zusammenfassend kann gesagt werden: Das Vorlesen macht Studierende stark, das Sprach- und Kulturbewusstsein im DaF-Bereich wird gefördert, die Kommunikation über die präsentierten Geschichten bzw. über die literarischen Texte wird unterstützt. Zudem kann man über Alltag und Ereignisse, aber auch über andere Themen sprechen - es bietet

\footnotetext{
${ }^{6}$ Vgl. Karacabey, Seval (im Druck): Steuerung des Literaturlesens mit dem Lesetagebuch. In: Uysal Ünalan, Saniye (Hrsg.): Ege Germanistik. Forschungen zur deutschen Sprache, Literatur und Kultur. Band 1. Izmir: Ege Üniversitesi Yayınları, o.S.
} 
Anlass zum Austausch. Darüber hinaus entlastet die Vorlesetätigkeit und bereichert zugleich den unterrichtlichen Alltag auch im Studium. Auf diese Weise führt es zu einem kontinuierlichen Austausch zwischen Lehrperson und Studierenden. Das Vorlesen kann besonders lebendig gestaltet werden und die Lernenden können besonders aktiv in das Vorlesen einbezogen werden:

- Die Vortragsweise dem Inhalt angepasst vorlesen, z. B. verärgert, pathetisch, befehlend usw.

- Einen Text mit Instrumenten oder akustischen Elementen vertonen.

- Zum Vorlesen/ Lesen eines Textes eine passende Hintergrundmusik suchen.

- Text durch Klären schwieriger, unbekannter Begriffe vorentlasten.

- Kurze, interessante Passagen zum Vorlesen auswählen, ggf. längere Texte kürzen. Pausen einlegen, wenn die Geschichte zu lang ist.

- Tätigkeiten vor, während oder nach dem Vorlesen beachten.

- Kurze epische Texte wie Kurzgeschichten, Balladen, Lieder vorziehen bzw. aus einem Buch kapitelweise vorlesen.

- Studierende zum selber vorlesen animieren oder dazu motivieren, sich gegenseitig Texte vorzulesen.

- Die Studierenden benötigen Zeit, um sich die Geschichte vorzustellen. Daher sollte Satz für Satz langsam vorgelesen werden.

- Reale Gegenstände sollten nach Möglichkeit miteinbezogen werden wie z. B. Puppen als Handlungsfiguren benutzen.

- Das Vorlesen/ Erzählen muss zum interaktiven Prozess werden, d. h. der Lehrer liest nicht nur vor, sondern involviert die Studierenden in den Erzählvorgang, u.a. indem er Fragen zu den vorgelesenen Teilen stellt.

\section{Fazit}

Das regelmäßige Vorlesen wirkt sich auf die Lernenenden positiv aus. Mit dem Vorlesen wird die Lesemotivation gesteigert und die Neugier auf Literatur unterstützt. Die Textauswahl muss durchdacht sein. Der Text soll lustig, interessant, ansprechend, leicht und spannend sein. Ebenfalls agieren Lehrpersonen als Lesermodelle, daher soll die Lehrperson auf die Betonung beim Vorlesen achten, verständlich vorlesen und Blickkontakt zu den Studierenden herstellen. Dabei spielen Mimik, Gestik, Stimme eine bedeutende Rolle. Das Vorlesen in der Fremdsprache kann im Anfänger- und Fortgeschrittenenbereich im Hochschulkontext eine lernfördernde Wirkung haben und als Lernaktivität bzw. Lesestrategie geübt werden. Allerdings ist die Bewusstmachung von Vorlesen als Lesestrategie, und dessen Rolle beim Fremdsprachenlerner immens. Vor allem leseungewohnte Fremdsprachenlerner könnten durch das Vorlesen den besonderen Wert von Literatur erfahren (Schönheit von Sprache, gezielter Einsatz sprachlicher Mittel, Dramatik von Sprache usw.), über Literatur reflektieren und sprechen und nicht zuletzt den Gefallen am Lesen in der Fremdsprache entdecken. Das Vorlesen und Hören von Geschichten ist im sprach- und literaturorientierten Fremdsprachenunterricht und beim Spracherwerb von nicht unerheblicher Bedeutung und sollte einen festen Platz im Unterricht einnehmen, weil durch Vorlesen an die Literalität herangeführt werden kann. 
Durch regelmäßiges Vorlesen können die Studierenden die Aussprache und die Betonung der Sprache trainieren und bekommen den Anstoß selber vorzulesen. Beim Vorlesen des Kinderbuches „Die Zugmaus“ geht es darum, den Studierenden Lesemotivation und den Mut zum Lesen fremdsprachiger Literatur $\mathrm{zu}$ vermitteln. Kinderromane sind zum Vorlesen empfehlenswert, da durch dieses Medium mehrere Kompetenzbereiche, wie die Lese- und Schreibkompetenz der Studierenden, gefördert werden. Eigene und fremde Lebenswelten können entdeckt und neue Perspektiven erkundet werden. Lernende sollten auch auf Hochschulebene mit dem Vorlesen in der Fremdsprache in Berührung kommen. In diesem Kontext können durch Kinderliteratur auch im Hochschulkontext geeignete Vorlesesituationen geschaffen werden. Die Bilder, die den Kinderroman begleiten, können Vorlesegespräche herbeiführen. Man kann einen literarischen Text unterschiedlich vortragen, z.B. das Vorlesen mit Fragen sprachfördernd gestalten. Das Stellen von Fragen zum Text ist eine bewährte Strategie. Es regt die Lernenden zum Nachdenken und zum Sprechen an und bietet ihnen die Möglichkeit, sich aktiv an der Interaktion mit dem Text zu beteiligen. Auch das "Feedback-Gespräch" und das „literarische Gespräch“ sind geeignete literarische Kommunikationsformen, die im Rahmen des fremdsprachlichen Vorlesens eingesetzt werden können. Studierende sollten für die besondere Qualität des Vorlesens sensibilisiert werden, weil sie selbst als Lehrpersonen agieren werden. Hierfür können auch Vorleseaktionen, Vorlesepraktiken gestaltet werden. Auch ehrenamtliche Vorlesepaten könnten das Vorlesen im Studienalltag nachhaltig stärken, sowie regelmäßige Vorlesestunden und Vorlesewettbewerbe könnten im Studium eingebaut werden. Allerdings sollte die Bedeutung bzw. Zweck des Vorlesens als Literacy-Aktivität den Deutschlehramtskandidaten bewusstgemacht werden und es sollten Anlässe bzw. „Anschlusskommunikation“ geschaffen werden, sprachlich zu handeln.

\section{Literaturverzeichnis}

Becker, Tabea / Müller, Claudia (2015): Vorlesen und Erzählen im Vergleich. In: Gressnich, Eva/ Müller, Claudia / Stark, Linda (Hg.): Lernen durch Vorlesen. Sprach- und Literaturerwerb in Familie, Kindergarten und Schule. Tübingen: Narr Francke Attempto Verlag, 77-93.

Belgrad, Jürgen / Schattmann, Isabell (2015): Vorlesen und Formen literaler Anschlusskommunikation. In: Zeitschrift Deutschunterricht: Vorlesen - Kulturtechnik, Verstehenshilfe, Zuhörtraining. Heft 6. Braunschweig: Westermann, 1-6.

Belgrad, Jürgen / Klippstein, Christin (2015): Leseförderung durch Vorlesen. Ein empirisch begründetes Plädoyer für das regelmäßige Vorlesen im Unterricht aller Schularten. In: Gressnich, Eva/ Müller, Claudia / Stark, Linda (Hg.): Lernen durch Vorlesen. Sprach- und Literaturerwerb in Familie, Kindergarten und Schule. Tübingen: Narr Francke Attempto Verlag, 180-198.

Ciepielewska-Kaczmarek, Luiza (2016): Einsatz von Kinterliteratur im frühen Fremdsprachenunterricht. Heft 2, 53. Jahrgang. In: Deutsch als Fremdsprache. Zeitschrift zur Theorie und Praxis des Faches Deutsch als Fremdsprache. Berlin: Erich Schmidt Verlag, 85-95.

Ergül, Cevriye / Akoğlu, Gözde / Sarıca, A. Dolunay / Tufan, Mümin / Karaman, Gökçe (2015): Ana Sınıflarında Gerçekleştirilen Birlikte Kitap Okuma Etkinliklerinin "Etkileşimli Kitap Okuma" bağlamında İncelenmesi. In: Mersin University Journal of the Faculty of Education 11(3), 603619. 
Gölitzer, Susanne (2010): Lesesozialisation. In: Lange, Günter / Weinhold, Swantje (Hg.): Grundlagen der Deutschdidaktik. Sprachdidaktik - Mediendidaktik - Literaturdidaktik. Baltmannsweiler: Schneider Verlag, 202-225.

Gressnich, Eva / Müller, Claudia / Stark, Linda (Hg.) (2015): Lernen durch Vorlesen. Sprach- und Literaturerwerb in Familie, Kindergarten und Schule. Tübingen: Narr Francke Attempto Verlag.

Güneş, Firdevs (2011): İlköğretimde Sesli Okumanın Önemi ve Yararları. Mili Eğitim. Sayı 191, 7-23.

Huneke, Hans-Werner / Steinig, Wolfgang (2002): Deutsch als Fremdsprache. Eine Einführung. 3., überarbeitete und erweiterte Auflage. Berlin: Erich Schmidt Verlag.

Karacabey, Seval (im Druck): Steuerung des Literaturlesens mit dem Lesetagebuch. In: Uysal Ünalan, Saniye (Hrsg.): Ege Germanistik. Forschungen zur deutschen Sprache, Literatur und Kultur. Band 1. Izmir: Ege Üniversitesi Yayınları, o.S.

Kuyumcu, Reyhan (2008): Mediennutzung zweisprachig aufwachsender Kinder mit Erstsprache Türkisch im häuslichen Umfeld und im Kindergarten. In: Wieler, Petra (Hg.): Medien als Erzählanlass. Wie lernen Kinder im Umgang mit alten und neuen Medien? 1. Auflage. Stuttgart: Fillibach bei Klett Verlag, 209-213.

Lundquist-Mog, Angelika / Widlok, Beate (2015): DaF für Kinder. 1. Auflage. Stuttgart: Ernst Klett Sprachen Verlag.

Nickel, Sven / Hering, Jochen (Hg.) (2007): Sprache und Literaturdidaktik im Elementarbereich. Literacy. In der Reihe Hochschuldidaktische Handreichungen. Universität Bremen. Download unter www.elementargermanistik.uni-bremen.de/Handreichung_Nickel_Literacy.pdf

Paefgen, Elisabeth K. (1999): Einführung in die Literaturdidaktik. Stuttgart: J. B. Metzler Verlag.

Rosebrock, Cornelia / Nix, Daniel (2012): Grundlagen der Lesedidaktik und der systematischen schulischen Leseförderung. 5., unveränderte Auflage. Baltmannsweiler: Schneider Verlag.

Timm, Uwe (2007): Die Zugmaus. 5. Auflage. München. Deutscher Taschenbuch Verlag.

\section{Internetquellen}

www.vds-ev-sachsen-anhalt.de (Letzter Zugriff: 30.07.2021).

https://www.familienservice.uni-mainz.de/files/2013/10/VLStudie_2015_Website_20151118_03.pdf (Letzter Zugriff: 10.10.2021).

https://www.familienservice.uni-mainz.de/files/2013/10/VLStudie_2015_Website_20151118_03.pdf (Letzter Zugriff: 30.09.2021).

www.nifbe.de (Letzter Zugriff: 15.10.2021). 